Published in final edited form as:

Sex Transm Dis. 2019 August ; 46(8): 493-501. doi:10.1097/OLQ.0000000000001010.

\title{
STD Partner Services Costs, other Resources, and Strategies across Jurisdictions to Address Unique Epidemic Characteristics and Increased Incidence
}

\author{
Rachel A. Silverman, PhD, ScM ${ }^{1}$, David A. Katz, PhD, MPH ${ }^{1,2}$, Carol Levin, PhD, MSc ${ }^{1}$, Teal \\ R. Bell, MPH ${ }^{3}$, Dawn Spellman, MA ${ }^{2}$, Lisa St. John, MBA, M.Ed ${ }^{4}$, Evelyn Manley Rodriguez ${ }^{5}$, \\ Matthew R. Golden, MD, MPH ${ }^{2,6}$, and Ruanne V. Barnabas, DPhil, MBChB, MSc ${ }^{1,6,7}$ \\ ${ }^{1}$ Department of Global Health, University of Washington, Seattle, WA, USA \\ ${ }^{2}$ Public Health - Seattle \& King County, Seattle, WA, USA \\ ${ }^{3}$ Washington State Department of Health, Olympia, WA, USA \\ ${ }^{4}$ Spokane Regional Health District, Spokane, WA, USA \\ ${ }^{5}$ Tacoma-Pierce County Health Department, Tacoma, WA, USA \\ ${ }^{6}$ Department of Medicine, University of Washington, Seattle, WA, USA \\ ${ }^{7}$ Department of Epidemiology, University of Washington, Seattle, WA, USA
}

\section{Abstract}

Background: STD partner services (PS) are a core component of STD programs. Data on costs are needed to support PS programming.

\begin{abstract}
Methods: In Washington State STD PS programs, disease intervention specialists (DIS) conduct telephone-based interviews and occasional field visits, offer expedited partner therapy (EPT) to heterosexuals with gonorrhea or chlamydia, and promote HIV testing, pre-exposure prophylaxis (PrEP), and HIV care. We conducted activity-based micro-costing of PS, including: observational and self-reported time studies and interviews. We analyzed cost, surveillance, and service delivery data to determine costs per program outcomes.
\end{abstract}

Results: In King, Pierce, and Spokane counties, respectively, DIS allocated 6.5, 6.4, and 28.8 hours per syphilis case and 1.5, 1.6, and 2.9 hours per gonorrhea/chlamydia case, on average. In 2016, each full-time DIS investigated 270, 268, and 61 syphilis and 1,177, 1,105, and 769 gonorrhea/chlamydia cases. $>80 \%$ of syphilis cases in King and Pierce were among men who have sex with men vs. $38 \%$ in Spokane. DIS spent $12 \%-39 \%$ of their time actively interviewing cases and notifying partners (clients), and the remaining time locating clients, coordinating and verifying care, and managing case reports. Time spent on EPT, HIV testing, and referrals to HIV treatment or PrEP, was minimal ( $<5$ minutes per interview) at locations with resources outside PS staff.

Corresponding author: Ruanne V. Barnabas, 325 Ninth Ave, Box 359927, Seattle, Washington, 98104, USA; phone, 206-520-3813; fax, 206-520-3831; rbarnaba@uw.edu.

Conflicts of interest: No authors have an association that might pose a conflict of interest to this study. 
Program cost-per-interview ranged from $\$ 527-\$ 2,210$ for syphilis, $\$ 219-\$ 484$ for gonorrhea, and \$164-\$547 for chlamydia.

Discussion: STD PS resource needs depended on epidemic characteristics and program models. Integrating HIV prevention objectives minimally impacted PS-specific program costs. Results can inform program planning, future budget impact, and cost-effectiveness analyses.

\section{Summary:}

Cost assessment of partner services for STDs found high variability in activities and costs across Washington State health jurisdictions. HIV prevention and treatment interventions had minimal impact on program costs.

\section{Keywords}

Partner Services; Bacterial sexually transmitted infections (Syphilis, Gonorrhea, Chlamydia); HIV; Costing

\section{Background}

The Centers for Disease Control and Prevention (CDC) recommends that health departments provide partner services (PS) to persons diagnosed with early syphilis, and some provide PS to selected individuals diagnosed with gonorrhea and chlamydial infection(1). PS generally involves health department staff interviewing infected persons (original patients [OPs]) to obtain information about their exposed sex partners to ensure that those partners are tested and treated appropriately(1). Beyond that, PS resources, practices, and objectives vary substantially(2-5). Some programs use PS as an HIV prevention intervention and have defined HIV testing, linkage and re-linkage of HIV positive persons to treatment, and referral of HIV-uninfected individuals - particularly men who have sex with men (MSM) to HIV pre-exposure prophylaxis (PrEP) as explicit PS outcomes(5-7).

Despite rising rates of bacterial STDs in the U.S.(8), CDC's annual STD budget declined 40\% between 2003 and 2017, adjusted for inflation(9). Understanding the costs of STD PS programs could help ensure that limited resources are used efficiently(10). Prior cost studies focus on either syphilis or gonorrhea, chlamydia and nongonococcal urethritis, or fail to capture heterogeneity between local jurisdictions or include HIV prevention objectives(1114).

Along with the U.S. as a whole, bacterial STD incidences are increasing throughout Washington State $(15,16)$. We described the epidemic characteristics and estimated the share of personnel time and financial costs associated with STD PS programs and activities at three high-burden local health jurisdictions that represent the geographic and resource diversity of Washington State. Results can inform both local providers and programs around the country. 


\section{Methods}

\section{Study Setting \& Summary}

In Washington State, STD PS interviews are primarily conducted over the telephone, integrate expedited partner therapy (EPT) for heterosexuals with gonorrhea or chlamydia(17), and promote HIV-specific interventions for MSM and transgender persons with an STD diagnosis or exposure. HIV interventions include HIV testing $(7,18)$, linkage to treatment(19), and linkage to PrEP for high-risk individuals(20). PS are provided to all cases of early syphilis and select cases of gonorrhea/chlamydia, including all pregnant women and persons with untreated infections, and in some cases, MSM, transgender persons, and adolescents depending on case risk factors and resource availability. Field visits were conducted on priority cases, only when efforts to reach the individual via several telephone and text attempts, emails, and letters had failed. Spokane also utilized Facebook messenger to make contact and conducted field visits when individuals with syphilis were incarcerated at the nearby local jail. King only provided field visits to untreated cases.

This study estimates the costs for STD PS delivery from the perspective of the health department at three local health jurisdictions: Public Health - Seattle \& King County (King), Tacoma-Pierce County Health Department (Pierce), Spokane Regional Health District (Spokane). These high-burden locations were selected to represent the diversity within the State. King serves the dense urban city of Seattle and the surrounding area, Pierce serves the city of Tacoma and the more rural surrounding area in the county directly south of King, and Spokane serves the less dense city of Spokane and the rural surrounding area in the eastern most part of the State. For each location, we present the population size reported by the U.S. census bureau and the number of reported STD cases by risk characteristic (MSM, men who have sex with women (MSW), men with unknown risk, and women) using county-level disease surveillance data from 2016. We conducted a micro-costing analysis in 2016-2017 to estimate staff time allocation for specific components of PS delivery and estimated the total time personnel allocated to STD PS. Analyses included a time study and financial expenditure assessment. We used surveillance and programmatic data to estimate health service delivery indicators, outcomes, and associated costs. Results are stratified by jurisdiction to investigate heterogeneity in disease burden, population characteristics, costs, and program models.

These activities were conducted as part of public health program evaluation and therefore not considered human subjects research. Analyses were conducted using Microsoft Excel 2017 and Stata/SE 14.2.

\section{Health Service Delivery Indicators}

We used county-level surveillance and PS data from 2016 to determine all health services indicators unless otherwise stated, including the total numbers of STD cases diagnosed, assigned for PS, and interviewed by DIS, and the numbers of partners named by OPs, notified by DIS or OP, tested, diagnosed, and treated following the OP's PS interview. We also determined the number of OPs provided with EPT, and HIV-negative clients (OPs and partners) tested for HIV and referred to PrEP through PS, referred to as enhanced PS 
outcomes. Information for PrEP referrals was collected empirically for OPs in King County and via DIS self-report for STD PS clients combined (OPs and partners) in Pierce and Spokane, so are not directly comparable. The number of HIV-infected OPs identified as inadequately engaged in HIV care (defined as not being on antiretroviral therapy or, if treatment status is unknown, reporting having no provider or visit in the prior 6 months/ scheduled future visit at the time of interview) and the number linked to care following PS (virally suppressed 6 months following interview) were estimated when available. Partner outcome indices were calculated by dividing the total number of partners with each outcome (number notified, tested, treated, etc.) by the number of OPs interviewed(21). Cases with multiple STDs (coinfections) were included as the higher priority STD in the following order: syphilis, gonorrhea, chlamydia.

\section{Time Study}

We conducted a time study of all DIS and other PS staff. First, an independent observer assessed how much time each staff member spent on each activity over several hours during a typical workday. To complement this information, each staff member was interviewed prior to the observations (and after if observations revealed omissions) to gain a more complete understanding of workflow, time allocation, and if they had unrelated job responsibilities; these data were used to estimate the proportion of administrative staff and DIS labor allocated to STD PS based on their self-report and confirmed by the supervisor. Second, PS staff conducted individual case tracking and reported specific activities from case report, assignment, through to case closure. Tracked cases were purposively sampled to include cases representing a variety of STDs and risk characteristics.

We estimated total personnel hours spent per week on key activities by STD. We also summarized tracking form data to present the means, medians, and variability in time spent per case by STD and population. The total time allocated per case was estimated by dividing the average weekly number of hours allocated for work related to STD PS by the average number of cases assigned to STD PS in a week (calculated by dividing the total number of cases worked in 2016 by 44 work weeks, the average number of weeks worked by staff in a year).

\section{Financial Expenditures Assessment}

2016 financial records for personnel salaries and benefits and overhead were adjusted based on the percentage of time staff allocated to STD PS to estimate the total STD PS program costs by STD type at each jurisdiction. Benefit costs were provided by the PS supervisor for each staff member and overhead costs were calculated as a percent of salaries, defined as the amount charged against labor in each county (33\% in King and 30\% in Pierce and Spokane). Activities and time unrelated to STD PS were excluded from analyses. The total costs per STD PS outcomes (e.g. OP interview, partner notified, tested and treated after DIS interview, etc.) were calculated by dividing the estimated total program cost by the number of individuals known to have received that service or have that outcome in 2016. Outcomes achieved per $\$ 100,000$ invested in STD PS were calculated by dividing the number of individuals known to have received that service by total program cost and multiplying by $\$ 100,000$. All costs were adjusted for inflation to 2016 US dollars. 


\section{Results}

\section{Workflow Summary}

At all three jurisdictions, administrative/data entry staff process laboratory and case reports for all gonorrhea/chlamydia cases. Once the initial case report is entered into the statewide web-based STD surveillance/PS database, cases are assigned to DIS for PS depending on STD, risk criteria, and available resources (Figure 1). For new syphilis cases, State Department of Health (DOH) staff assign possible cases to DIS for review and PS.

Observations were conducted on select days in 2016-2017 in each health department. Preassignment administrative and data entry work for gonorrhea/chlamydia was conducted by three staff members in King and one each in Pierce and Spokane. There were 7, 5, and 4 DIS working on STD PS in King, Pierce, and Spokane, respectively (see supplement for additional details on staff time).

\section{Time Study}

In King, Pierce, and Spokane, respectively, DIS combined spent an average total of 73, 17, and 73 hours per week on syphilis PS overall; worked an average of 11,3, and 3 cases per week; and allocated an average of 6.5, 6.4, and 28.8 hours per case (Figure 2). DIS combined spent an average total of 142,88 , and 27 hours per week on gonorrhea/chlamydia PS work overall; worked an average of 96, 55, and 9 cases per week; and allocated an average of $1.5,1.6$, and 2.9 hours per case, respectively.

Estimated aggregate time in hours and percent of time spent per week on PS activities varied across STD and jurisdiction, but some trends were observed (Supplemental Figure 1 and Supplemental Table 1). Administrative staff's work was similar for all reported gonorrhea/ chlamydia cases regardless of whether the case met criteria for PS follow-up and DIS investigation, spending the majority of their time on data entry at all jurisdictions ( $>53 \%)$. King administrative staff and DIS spent the largest percentage of time (17\% and 9\%, respectively) contacting healthcare providers to obtain and complete case reports vs. Pierce and Spokane (<3\%). For syphilis STD PS, DIS spent a similar share of time contacting healthcare providers in King (22\%) and Pierce (19\%) vs. less time in Spokane (3\%). Spokane staff directly accessed electronic medical records (EMR) and could look up case information directly.

For syphilis, DIS spent $6 \%, 31 \%$, and $11 \%$ of time conducting case interviews and partner notification vs. $21 \%, 39 \%$, and $16 \%$ of gonorrhea/chlamydia work-time in King, Pierce, and Spokane, respectively. DIS in Spokane spent a greater proportion of time conducting field work for gonorrhea/chlamydia (18\% vs. $<6 \%$ in King and Pierce) and reported interviews were often conducted at the nearby county detention center. DIS working on gonorrhea/ chlamydia in Spokane and Pierce spent a greater percent of time using electronic resources (e.g. EMRs and Facebook) to locate and contact OPs and partners than in King. Facebook was generally used after other methods (e.g. phone calls, texting) were unsuccessful. Only DIS in Spokane frequently used local police blotters. In all jurisdictions, not all staff had access to commonly used electronic data sources (e.g. Accurint ${ }^{\circledR}$ or the state syphilis serology database), resulting in interruptions to staff with access to obtain information. 
Time spent directly on casework captured by individual case tracking varied substantially across cases (Supplemental Table 2). More DIS time was spent on syphilis than gonorrhea/ chlamydia casework across jurisdictions. Among cases with substance use information, casework for methamphetamine users took longer than non-users for Spokane syphilis cases (limited data regarding methamphetamine use was available from tracked cases in King and Pierce). While complicated high-priority cases like neurological and neonatal syphilis are rare, DIS spent a much larger amount of time conducting related casework, resulting in extreme outliers for time spent per case.

STD PS objectives such as EPT, HIV testing, linkage and re-linkage to HIV care, and PrEP referrals took minimal time relative to overall work (30 seconds to 2 min per topic per interview), except for PrEP referrals in Pierce. Clients often already had a documented HIV test, were not HIV-positive, or were already linked to care and on antiretroviral treatment if they were HIV-positive, minimizing additional DIS interventions. Some PrEP eligible clients had already discussed PrEP with their healthcare provider. King and Spokane had public health PrEP coordinators so DIS typically took only a few minutes to assess client eligibility and interest in PrEP and then referred the client to the coordinator. However, DIS in Pierce coordinated PrEP referrals and initiations themselves, including counseling and collecting blood samples for PrEP eligibility required for initiation, which DIS reported took approximately 69-155 minutes per referral for 3-6 clients per week.

\section{Disease Burden, Characteristics, and Partner Services Outcomes}

King has the largest population and most reported STD diagnoses (Table 1). Pierce had approximately twice the number of reported gonorrhea/chlamydia cases than Spokane, but a similar number of syphilis cases. The majority (>80\%) of syphilis cases in King and Pierce were identified as MSM vs. 38\% in Spokane. For gonorrhea, about half (52\%) of cases in King were identified as MSM vs. $<20 \%$ in Pierce and Spokane.

Nearly all (>96\%) of reported syphilis cases, $66 \%-95 \%$ of reported gonorrhea cases, and a minority of chlamydia cases were assigned to PS across the jurisdictions (Table 2).

Approximately 270, 268, and 61 syphilis and 1,177, 1,105, and 769 gonorrhea/chlamydia cases were assigned for PS per full-time DIS, in King, Pierce and Spokane, respectively. Of reported syphilis cases, 70\%-91\% were successfully interviewed by DIS and 35\%-70\% of reported gonorrhea cases were successfully interviewed (Table 3a). Partner outcome indices were lowest in King for syphilis cases and relatively similar across jurisdictions for gonorrhea/chlamydia. In 2016, median time from lab diagnosis to DIS interview in King, Pierce and Spokane, respectively, for syphilis was 13 days (IQR 6-32), 5 (1-12), and 5 (0-13), and for gonorrhea/chlamydia was 8 (3-18), 4 (0-10), and 8 (4-20).

\section{Financial Expenditures and Costs per Outcome}

We estimated that the annual costs for STD PS were $\$ 798,141, \$ 416,098$, and $\$ 400,759$ in King, Pierce, and Spokane, respectively (Table 2). At the time of observations, syphilisrelated work accounted for an estimated 28\%, 15\%, and 57\% of total STD PS resources, respectively. Spokane was uniquely supported by an on-site DOH-funded syphilis consultant (costs were included in estimate as the consultant's contributions were necessary for service 
delivery to address the epidemic at this location). The incremental costs of PS activities can be derived by multiplying the program costs (Table 2) by the percent of personnel-time spent on activities (Supplemental Table 1).

The estimated total costs per OP interview were lowest in Pierce and highest in Spokane for all STDs, and higher for syphilis vs. gonorrhea/chlamydia (Table 3a). The total cost per HIV test of all STD PS clients combined following the DIS interview ranged from $\$ 988-\$ 5,467$ (Table 3b), per client newly diagnosed with HIV was $>\$ 130,000$ for all jurisdictions, per OP identified as HIV infected and inadequately engaged in care ranged from $\$ 10,203-\$ 111,837$, and per HIV-uninfected client accepting a PrEP referral ranged from $\$ 2,551-\$ 57,467$. Outcomes achieved per $\$ 100,000$ invested in STD PS varied substantially by jurisdiction (Table 3c). For syphilis and gonorrhea, more outcomes were achieved in Pierce per $\$ 100,000$ invested than in King and Spokane.

\section{Discussion}

Our study describes the nuances of STD PS work and the costs associated with service delivery. Our findings can be used to implement and improve similar programs in order to best address STD epidemics with limited resources. We found large variability in the time and cost required to provide PS to clients with different STDs across local health jurisdictions in Washington State, which was dependent on STI epidemiologic characteristics and available support services. Program cost-per-interview ranged from \$527$\$ 2,210$ for syphilis, $\$ 219-\$ 484$ for gonorrhea, and $\$ 164-\$ 547$ for chlamydia. Our study found similar trends in the relative costs between STDs compared to a similar, state-level, costing analysis of PS in New York in 2014(13,22) that estimated their cost per interview was $\$ 1,072, \$ 608, \$ 635$, for syphilis, gonorrhea, and chlamydia, respectively(13). In our study, the additional resources required for syphilis PS were partially explained by the work exclusively being performed by highly-trained DIS compared to administrative staff.

Syphilis PS consistently involved greater resources than gonorrhea/chlamydia, and heterosexual syphilis cases were consistently more resource-intensive than MSM. The cost per syphilis case assigned in Spokane was approximately four times that in King and Pierce. Most syphilis cases in Spokane were among heterosexuals, many of whom were suspected methamphetamine users, compared to King and Pierce where cases were primarily among MSM. Spokane DIS also spent a greater proportion of time in the field compared to King and Pierce. The higher costs per case, observed in Spokane largely reflects epidemic characteristics and inherent difficulties engaging a highly socially marginalized and highpriority heterosexual population. Health departments often place higher priority on syphilis cases due to the greater morbidity associated with this infection. Syphilis is especially concerning among heterosexuals and pregnant women given the risk and severity of congenital syphilis, which requires immediate DIS response and a large allocation of staff resources and time. Syphilis rates among heterosexuals in the US are now rising(8), which could increase the cost of PS.

Our findings highlight opportunities for improving the efficiency of PS. We observed that the majority of DIS time was spent on data entry and searching for clients prior to 
successfully interviewing cases and contacting partners at all locations. More efficient surveillance mechanisms could help providers and laboratories complete case-reporting in a timely manner resulting in faster PS responses, less time spent contacting providers, collecting data, and on data entry, and more time spent on casework. An electronic reporting system to submit and view case reports, as well as full access to EMR, would greatly reduce the burden on PS staff and healthcare providers. Additionally, increased access to restricted databases (e.g. Accurint ${ }^{\circledR}$ ) and better data sharing between the state DOH and across neighboring jurisdictions could facilitate faster casework.

Adding HIV-related services was generally not time-consuming relative to overall work and resulted in substantial numbers of MSM accepting referrals for PrEP in King and Pierce. PrEP referrals required substantially more DIS resources in Pierce, which lacked dedicated PrEP referral coordinators. The total STD PS program cost per HIV test following PS intervention found in our study $(\$ 998-\$ 5,467)$ was within the range of estimates of costs per partners tested in HIV PS programs in studies conducted in other locations across the US(23-25). Seattle has achieved the UNAIDS goal of 90-90-90(26), which likely contributed to the high cost per new HIV diagnosis following STD PS intervention $(\$ 133,586-\$ 416,098)$ in our study compared to other programs in the US(13, 23, 24, 27). In contrast to the high total cost per HIV diagnosis, the total cost per client accepting a PrEP referral in our study ranged from $\$ 2,551-\$ 57,467$ for syphilis and $\$ 3,726-\$ 6,118$ for gonorrhea/chlamydia cases.

The low incremental cost associated with adding HIV interventions to STD PS should prompt health departments to integrate them into existing programs. While HIV case-finding and the identification of out-of-care PWH were rare outcomes in our STD PS programs (and thus had a high cost per outcome), this was likely due to low frequencies of undiagnosed and untreated HIV cases within these jurisdictions(28), and other areas may have very different experiences. HIV prevention benefits can help justify expanding STD PS resources for the explicit purpose of HIV case-finding. Whether program costs are a good value requires costeffectiveness analyses incorporating the various outcomes measured.

This study has several strengths. We conducted direct observations and tracked individual cases across multiple health jurisdictions across Washington State with different epidemic characteristics, program models, and resources. Study limitations include that STD PS work vary over time and are not captured well by short observation periods. In addition, though efforts were made to minimize imposition of the time study itself, staff likely work differently while being observed(29). We attempted to address this by triangulating data from interviews, case-tracking forms, and observations. Additionally, the estimates of cases interviewed and partners notified derived from the time study were similar to the empirical case counts from surveillance that we used in our analysis. Not all partners treated after DIS interview may have been the result of DIS intervention, as some partners may have sought treatment regardless(30). These data may not be generalizable to programs in different settings. However, the detailed findings may be informative to any health department program. 
Our results can inform the operational costs, areas to improve efficiency, and budget impact of STD PS in U.S. health jurisdictions. Given that the lifetime cost of one HIV case is estimated to be $\$ 400,000(31)$, efforts to integrate interventions to prevent HIV transmission within STD PS may be highly cost-effective. A cost-effectiveness analysis of STD PS that will balance incurred costs against savings from HIV and STD prevention is needed.

\section{Supplementary Material}

Refer to Web version on PubMed Central for supplementary material.

\section{Acknowledgements:}

We are grateful to the STD partner services staff at Public Health - Seattle \& King County, Tacoma-Pierce County Health Department, Spokane Regional Health District for their participation in this study. We also thank the Health Economic Study Team (HEIST) at the University of Washington.

Funding: This work was supported by the U.S. Centers for Disease Control and Prevention [H25PS004364 and H25PS005108] and by the University of Washington / Fred Hutch Center for AIDS Research (CFAR), an NIHfunded program under award number AI027757 which is supported by the following NIH Institutes and Centers: NIAID, NCI, NIMH, NIDA, NICHD, NHLBI, NIA, NIGMS, NIDDK. The content is solely the responsibility of the authors and does not necessarily represent the official views of the National Institutes of Health.

\section{References}

1. Centers for Disease Control and Prevention. Recommendations for partner services programs for HIV infection, syphilis, gonorrhea, and chlamydial infection. MMWR Recomm Rep 2008;57(RR-9):1-83; quiz CE1-4.

2. Desir FA, Ladd JH, Gaydos CA. Survey of partner notification practices for sexually transmissible infections in the United States. Sex Health. 2016;13(2):162-9. [PubMed: 26841251]

3. Hogben M, Collins D, Hoots B, O'Connor K. Partner Services in Sexually Transmitted Disease Prevention Programs: A Review. Sex Transm Dis 2016;43(2 Suppl 1):S53-62. [PubMed: 26779688]

4. Golden MR, Hogben M, Handsfield HH, St Lawrence JS, Potterat JJ, Holmes KK. Partner notification for HIV and STD in the United States: low coverage for gonorrhea, chlamydial infection, and HIV. Sex Transm Dis 2003;30(6):490-6. [PubMed: 12782949]

5. Golden MR, Katz DA, Dombrowski JC. Modernizing Field Services for Human Immunodeficiency Virus and Sexually Transmitted Infections in the United States. Sex Transm Dis 2017;44(10):599607. [PubMed: 28876325]

6. Katz DA, Dombrowski JC, Bell TR, Kerani RP, Golden MR. HIV Incidence Among Men Who Have Sex With Men After Diagnosis With Sexually Transmitted Infections. Sex Transm Dis 2016;43(4): 249-54. [PubMed: 26967302]

7. Katz DA, Dombrowski JC, Kerani RP, et al. Integrating HIV Testing as an Outcome of STD Partner Services for Men Who Have Sex with Men. AIDS Patient Care STDS. 2016;30(5):208-14. [PubMed: 27158848]

8. Centers for Disease Control and Prevention. Sexually Transmitted Disease Surveillance 2016 Atlanta: U.S. Department of Health and Human Services; 2017.

9. National Center for HIV/AIDS VH, STD, and TB Prevention (NCHHSTP) Newsroom,. Telephone Press Briefing: New CDC analysis shows steep and sustained increases in STDs. David Harvey, MSW, Executive Director National Coalition of STD Directors (NCSD). Telebriefing Transcript: 2018 STD Prevention Conference Accessed at https:/www.cdc.gov/nchhstp/newsroom/2018/pressbriefing-transcript-2018-std-prevention-conf.html 2018.

10. Rabarison KM, Bish CL, Massoudi MS, Giles WH. Economic Evaluation Enhances Public Health Decision Making. Front Public Health. 2015;3:164. [PubMed: 26157792] 
11. Rahman MM, Khan M, Gruber D. A Low-Cost Partner Notification Strategy for the Control of Sexually Transmitted Diseases: A Case Study From Louisiana. Am J Public Health. 2015;105(8): 1675-80. [PubMed: 25689204]

12. Katz BP, Danos CS, Quinn TS, Caine V, Jones RB. Efficiency and cost-effectiveness of field follow-up for patients with Chlamydia trachomatis infection in a sexually transmitted diseases clinic. Sex Transm Dis 1988;15(1):11-6. [PubMed: 3358237]

13. Johnson BL, Tesoriero J, Feng W, Qian F, Martin EG. Cost Analysis and Performance Assessment of Partner Services for Human Immunodeficiency Virus and Sexually Transmitted Diseases, New York State, 2014. Health Serv Res 2017;52 Suppl 2:2331-42. [PubMed: 28799163]

14. Reynolds SL, Kapadia AS, Leonard L, Ross MW. Examining the direct costs and effectiveness of syphilis detection by selective screening and partner notification. J Public Health Med 2001;23(4): 339-45. [PubMed: 11873899]

15. Assessment Unit Office of Infectious Disease Disease Control and Health Statistics WSDoH. STD Fast Facts: Washington State 2017. 2018.

16. Assessment Unit Office of Infectious Disease Disease Control and Health Statistics WSDoH. STD Fast Facts: Washington State 2016. 2018.

17. Golden MR, Kerani RP, Stenger M, et al. Uptake and population-level impact of expedited partner therapy (EPT) on Chlamydia trachomatis and Neisseria gonorrhoeae: the Washington State community-level randomized trial of EPT. PLoS Med 2015;12(1):e1001777. [PubMed: 25590331]

18. Katz DA BT, Dombrowski JC, Kerani RP, Aubin MR, Bryan Z, Kern DA, Heal DD, Golden MR. Sexually Transmitted Disease Partner Services Increases HIV Testing among Partners of Men Who Have Sex with Men. STD Prevention Conference Atlanta, GA; 2016.

19. Katz DA GM, Bell TR, Kerani RP, Aubin MR, Bryan Z, Kern DA, Heal DD, Dombrowski JC. Using Sexually Transmitted Disease Partner Services to Promote Engagement in HIV Care among Persons Living with HIV. STD Prevention Conference Atlanta, GA; 2016.

20. Katz DA DJ, Barry M, Spellman D, Bell TR, Golden MR. STD Partner Services to Monitor and Promote HIV Pre-Exposure Prophylaxis Use among Men Who Have Sex with Men. JAIDS. In press.

21. Heumann CL, Katz DA, Dombrowski JC, Bennett AB, Manhart LE, Golden MR. Comparison of In-Person Versus Telephone Interviews for Early Syphilis and Human Immunodeficiency Virus Partner Services in King County, Washington (2010-2014). Sex Transm Dis 2017;44(4):249-54. [PubMed: 28282653]

22. Martin EG, Feng W, Qian F, Johnson B. Delivering Partner Services to Reduce Transmission and Promote Linkage to Care: Process Outcomes Varied for Chlamydial Infection, Gonorrhea, HIV, and Syphilis Cases. J Public Health Manag Pract 2017;23(3):242-6. [PubMed: 26480283]

23. Li XC, Kusi L, Marak T, Bertrand T, Chan PA, Galarraga O. The Cost and Cost-utility of Three Public Health HIV Case-finding Strategies: Evidence from Rhode Island, 2012-2014. AIDS Behav 2017.

24. Shrestha RK, Begley EB, Hutchinson AB, et al. Costs and effectiveness of partner counseling and referral services with rapid testing for HIV in Colorado and Louisiana, United States. Sex Transm Dis 2009;36(10):637-41. [PubMed: 19955875]

25. Zulliger R, Maulsby C, Solomon L, et al. Cost-utility of HIV Testing Programs Among Men Who Have Sex with Men in the United States. AIDS Behav 2017;21(3):619-25. [PubMed: 27624729]

26. HIV/AIDS Epidemiology Unit Public Health - Seattle \& King County and the Infectious Disease Assessment Unit - Washington State Department of Health. HIV/AIDS Epidemiology Report 2017, Volume 862017.

27. Huang YL, Lasry A, Hutchinson AB, Sansom SL. A systematic review on cost effectiveness of HIV prevention interventions in the United States. Appl Health Econ Health Policy. 2015;13(2): 149-56. [PubMed: 25536927]

28. HIV/AIDS Epidemiology Unit - Public Health - Seattle \& King County, Infectious Disease Assessment Unit - Washington State Department of Health. HIV/AIDS Epidemiology Report. 2016; Volume 85. 
29. Chen LF, Vander Weg MW, Hofmann DA, Reisinger HS. The Hawthorne Effect in Infection Prevention and Epidemiology. Infect Control Hosp Epidemiol 2015;36(12):1444-50. [PubMed: 26383964]

30. Dombrowski JC, Hughes JP, Buskin SE, et al. A Cluster Randomized Evaluation of a Health Department Data to Care Intervention Designed to Increase Engagement in HIV Care and Antiretroviral Use. Sex Transm Dis 2018;45(6):361-7. [PubMed: 29465679]

31. Centers for Disease Control and Prevention 2017;Pages. Accessed at https://www.cdc.gov/hiv/ programresources/guidance/costeffectiveness/index.html Accessed Feb 2, 2018. 


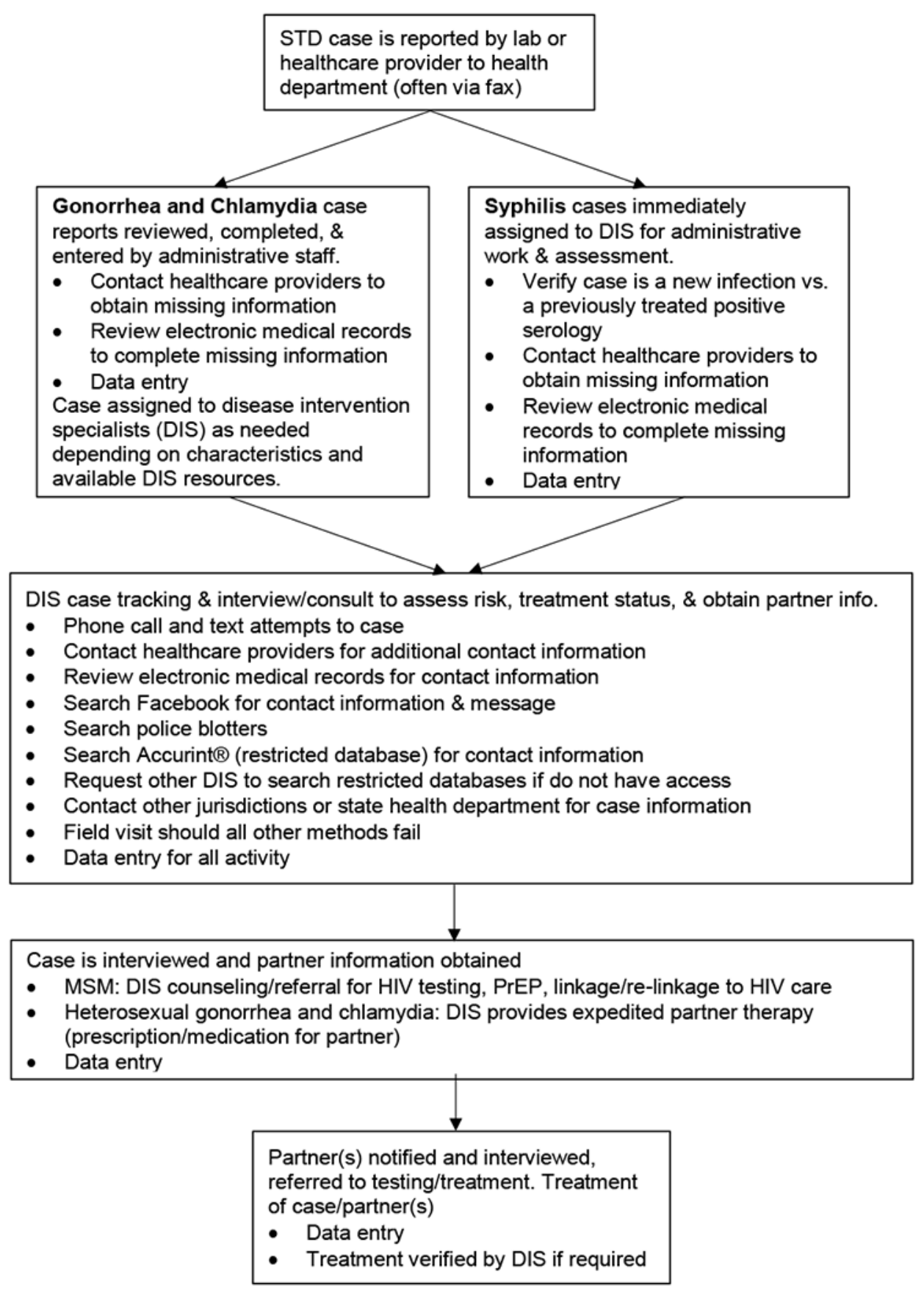

Figure 1.

Flow Chart of STD Partner Services Activities 


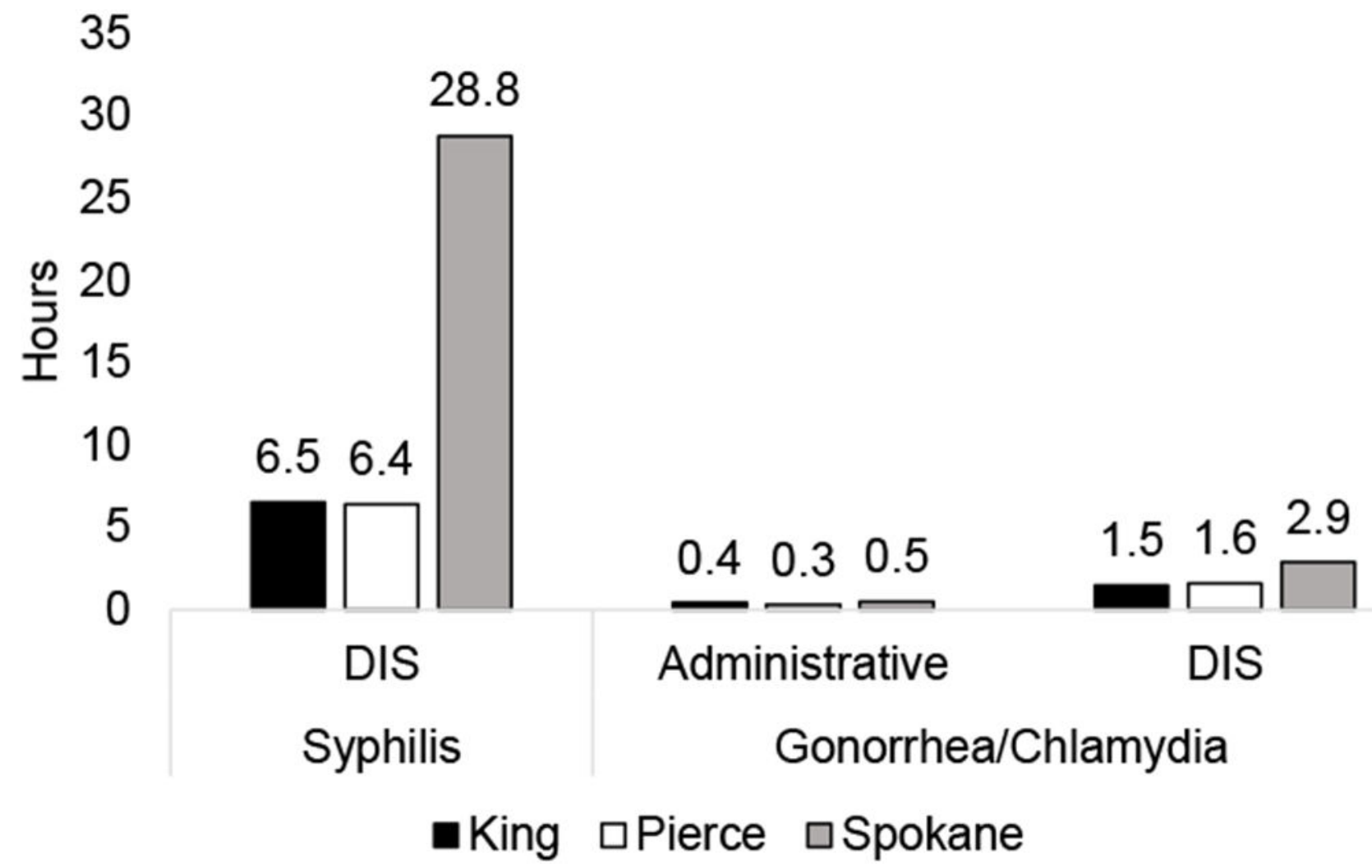

Figure 2.

Total hours per case by STD and type of staff

*Calculated by dividing the combined employee time spent on each STD (as reported in staff interviews) by the number of cases assigned for each jurisdiction in 2016.

Administrative work is performed on all reported gonorrhea and chlamydia cases, a selection of which are assigned to DIS for partner services based on risk criteria and resource availability. All reported syphilis cases are immediately assigned to DIS for administrative work, review, and partner services. 
Table 1.

Population Size and Reported STD Case Risk Characteristics in 2016

\begin{tabular}{llccc}
\hline Category & King & Pierce & Spokane \\
\hline Total Population Estimate & ${ }^{a}$ & $2,149,970$ & 861,312 & 499,072 \\
\hline Reported Cases by STD \& Risk Characteristic & & & \\
\hline \multirow{5}{*}{ Early syphilis } & Total & $516(100 \%)$ & $116(100 \%)$ & $114(100 \%)$ \\
& MSM & $455(88 \%)$ & $95(82 \%)$ & $43(38 \%)$ \\
& MSW & $20(4 \%)$ & $10(9 \%)$ & $37(32 \%)$ \\
& Men (unknown) & $24(5 \%)$ & $3(3 \%)$ & $1(1 \%)$ \\
& Women & $17(3 \%)$ & $8(7 \%)$ & $33(29 \%)$ \\
\hline \multirow{5}{*}{ Gonorrhea } & Total & $3,378(100 \%)$ & $1,181(100 \%)$ & $511(100 \%)$ \\
& MSM & $1,770(52 \%)$ & $214(18 \%)$ & $79(15 \%)$ \\
& MSW & $544(16 \%)$ & $346(29 \%)$ & $137(27 \%)$ \\
& Men (unknown) & $255(8 \%)$ & $98(8 \%)$ & $67(13 \%)$ \\
& Women & $809(24 \%)$ & $523(44 \%)$ & $228(45 \%)$ \\
\hline \multirow{5}{*}{ Chlamydia } & Total & $8,627(100 \%)$ & $4,610(100 \%)$ & $2,306(100 \%)$ \\
& MSM & $1,486(17 \%)$ & $138(3 \%)$ & $66(3 \%)$ \\
& MSW & $1,520(18 \%)$ & $933(20 \%)$ & $368(16 \%)$ \\
& Men (unknown) & $666(8 \%)$ & $431(9 \%)$ & $231(10 \%)$ \\
& Women & $4,953(57 \%)$ & $3,108(67 \%)$ & $1,641(71 \%)$ \\
\hline
\end{tabular}

Abbreviations: STD, sexually transmitted disease; MSM, men who have sex with men (includes those who also have sex with women); MSW, men who have sex with women

${ }^{a}$ Estimated by the US census bureau as of July 1, 2016 
Table 2.

2016 Annual Financial Expenditures ${ }^{a}$ of the STD Partner Services Program and Total Expenditures by STD

\begin{tabular}{|c|c|c|c|}
\hline Category & King & Pierce & Spokane \\
\hline Number of OPs assigned to DIS & 4,701 & 2,359 & 631 \\
\hline Early syphilis & 493 & 114 & 112 \\
\hline Gonorrhea & 2,848 & 1,119 & 339 \\
\hline Chlamydia & 1,486 & 1,126 & 180 \\
\hline Full-Time DIS providing STD PS $(40 \mathrm{hr} / \mathrm{wk})$ & 5.4 & 2.6 & 2.5 \\
\hline Early syphilis & 1.8 & 0.4 & 1.8 \\
\hline Gonorrhea/Chlamydia & 3.6 & 2.2 & 0.7 \\
\hline \multicolumn{4}{|l|}{ Number of OPs assigned per DIS } \\
\hline Early syphilis & 270 & 268 & 61 \\
\hline Gonorrhea/chlamydia & 1,177 & 1,105 & 769 \\
\hline Full-Time administrative staff for STD PS ${ }^{b}$ & 1 & 0.4 & 0.3 \\
\hline Total salary $\&$ benefits for STD PS staff & $\$ 643,098(81 \%)$ & $\$ 342,163(82 \%)$ & $\$ 327,757(82 \%)$ \\
\hline Overhead & $\$ 149,736(19 \%)$ & $\$ 73,935(18 \%)$ & $\$ 70,738(18 \%)$ \\
\hline Salary, benefits, overhead total & $\$ 792,834(100 \%)$ & $\$ 416,098(100 \%)$ & $\$ 398,495(100 \%)$ \\
\hline Administrative & $\$ 107,261(14 \%)$ & $\$ 35,738(9 \%)$ & $\$ 25,528(6 \%)$ \\
\hline DIS & $\$ 633,140(80 \%)$ & $\$ 360,193(87 \%)$ & $\$ 275,561(69 \%)$ \\
\hline Supervisor & $\$ 45,588(6 \%)$ & $\$ 20,167(5 \%)$ & $\$ 97,405(24 \%)$ \\
\hline Epidemiology support & $\$ 6,845(1 \%)$ & N/A & N/A \\
\hline Vehicle \& fuel & $\$ 5,306(0.7 \%)$ & N/A & $\$ 2,262(0.6 \%)$ \\
\hline Total & $\$ 798,141(100 \%)$ & $\$ 416,098(100 \%)$ & $\$ 400,759(100 \%)$ \\
\hline \multicolumn{4}{|l|}{$\underline{\text { Total expenditures by STD }}^{c}$} \\
\hline Early syphilis & $\$ 220,576(28 \%)$ & $\$ 51,017(12 \%)$ & $\$ 229,869(57 \%)$ \\
\hline Gonorrhea & $\$ 391,089(49 \%)$ & $\$ 179,866(43 \%)$ & $\$ 111,837(28 \%)$ \\
\hline Chlamydia & $\$ 186,475(23 \%)$ & $\$ 185,215(45 \%)$ & $\$ 59,053(15 \%)$ \\
\hline
\end{tabular}

Abbreviations: STD, sexually transmitted disease; PS, partner services; OP, original patient; DIS, disease intervention specialist; N/A, not available

Includes only staff costs for work on cases that receive STD PS (costs for data entry and other work on cases that are not assigned to STD PS or otherwise followed by DIS are excluded)

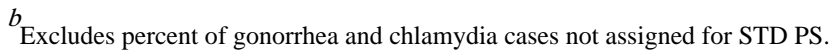

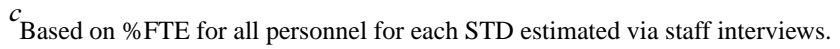




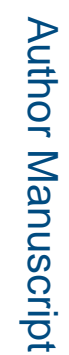

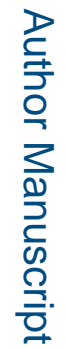

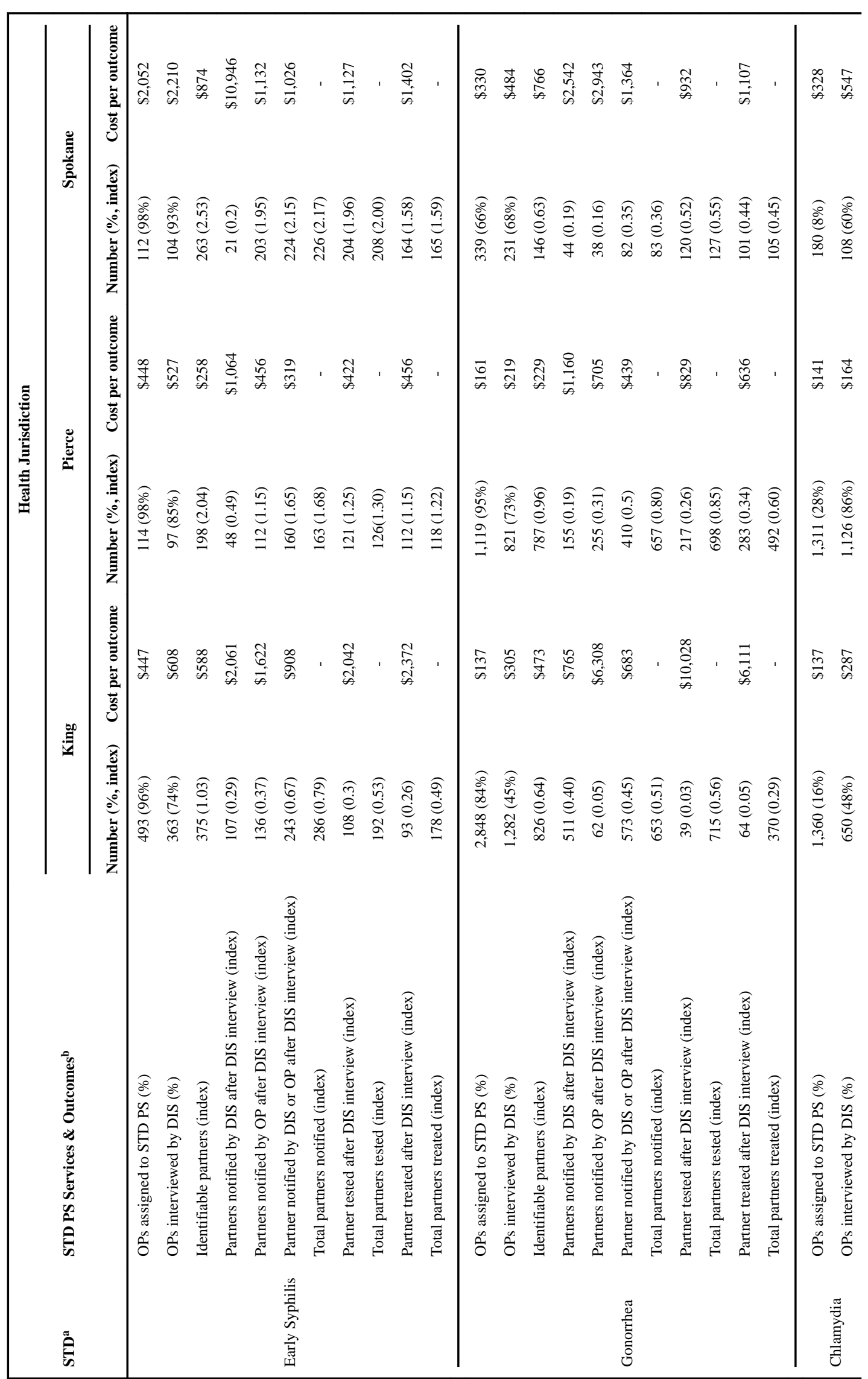

Sex Transm Dis. Author manuscript; available in PMC 2020 August 01. 


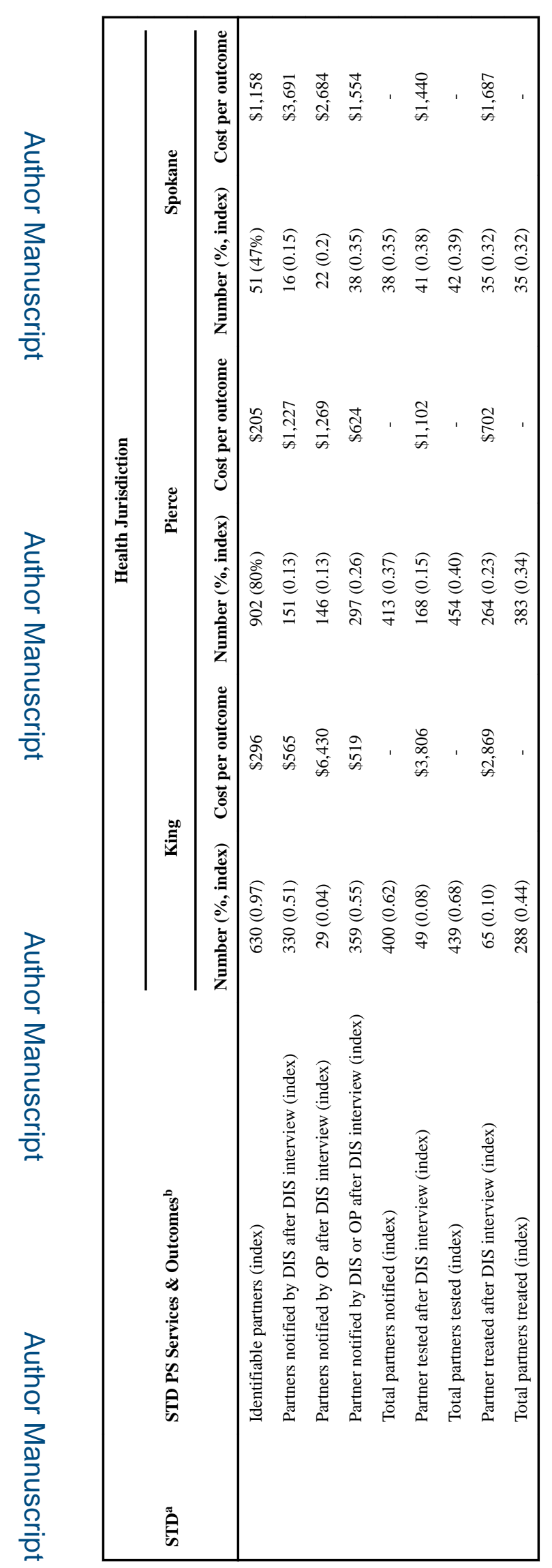




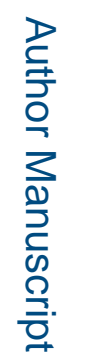

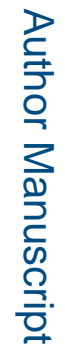

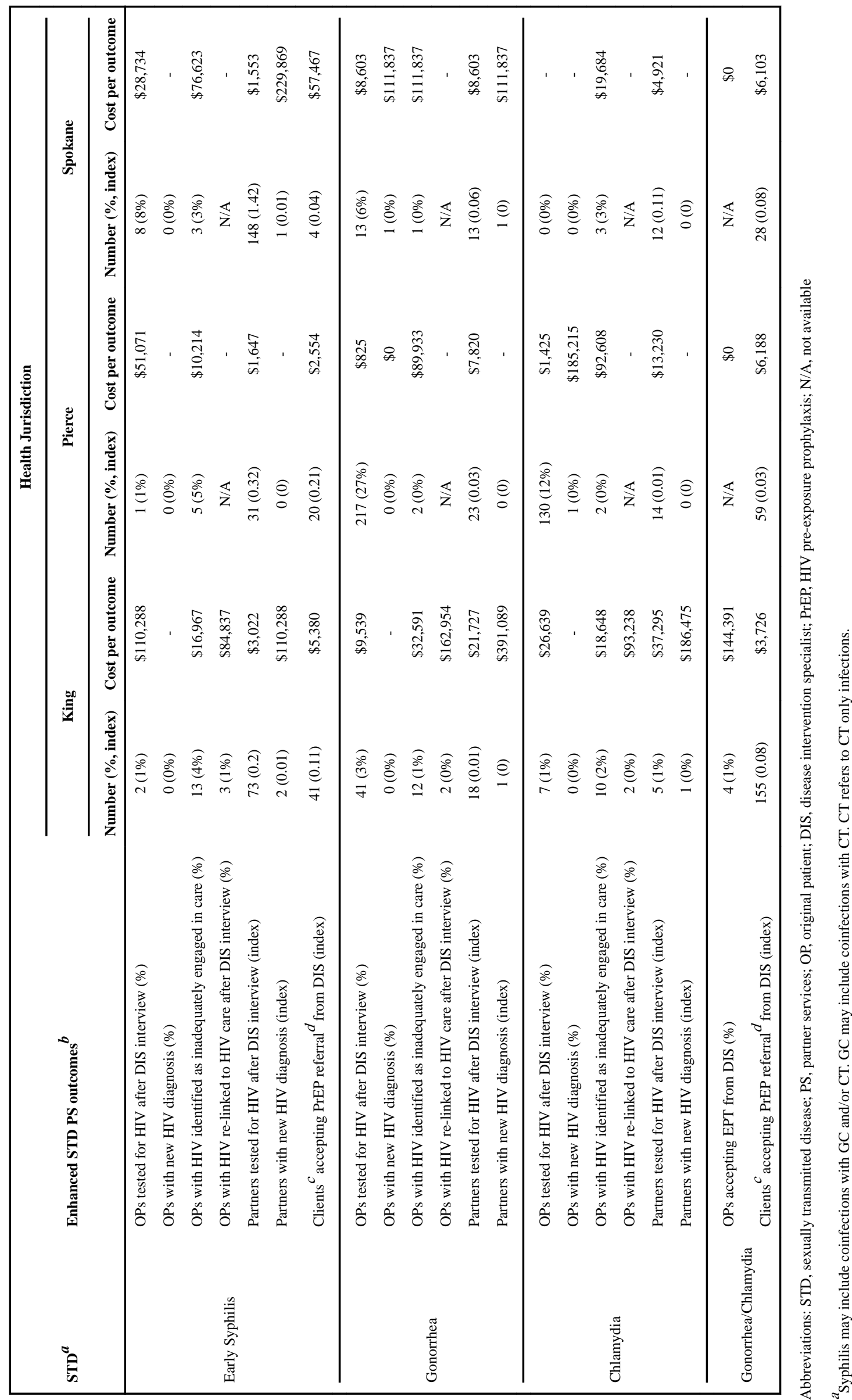




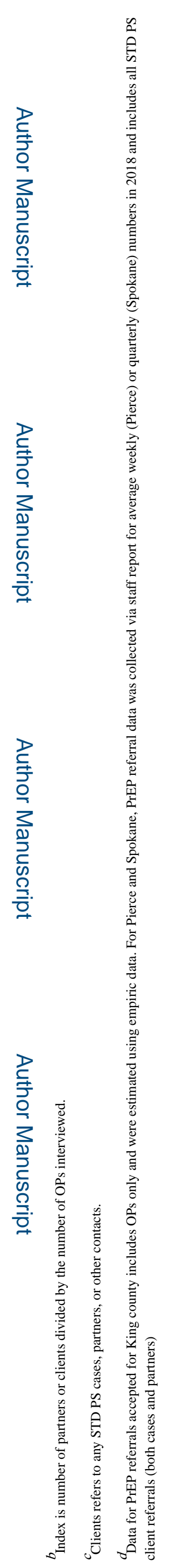

Sex Transm Dis. Author manuscript; available in PMC 2020 August 01. 
Table 3c.

Select STD Partner Services Outcomes per $\$ 100,000$

\begin{tabular}{|c|c|c|c|c|}
\hline STD & Outcome & King & Pierce & Spokane \\
\hline \multirow{6}{*}{ Early syphilis $^{a}$} & Partners notified by DIS or OP after interview & 110 & 314 & 97 \\
\hline & Partners tested after interview & 49 & 237 & 89 \\
\hline & Partners treated after interview & 42 & 220 & 71 \\
\hline & Clients $^{b}$ with new HIV diagnosis & 1 & 0 & 0 \\
\hline & OPs re-linked to HIV care & 1 & - & - \\
\hline & PrEP referrals accepted ${ }^{c}$ & 19 & 39 & 2 \\
\hline \multirow{5}{*}{ Gonorrhea } & Partners notified by DIS or OP after interview & 147 & 228 & 73 \\
\hline & Partners tested after interview & 10 & 121 & 107 \\
\hline & Partners treated after interview & 16 & 157 & 90 \\
\hline & Clients $^{b}$ with new HIV diagnosis & 0 & 0 & 2 \\
\hline & OPs re-linked to HIV care & 1 & - & - \\
\hline \multirow{5}{*}{ Chlamydia } & Partners notified by DIS or OP after Interview & 193 & 160 & 64 \\
\hline & Partners tested after interview & 26 & 91 & 69 \\
\hline & Partners treated after interview & 35 & 143 & 59 \\
\hline & Clients $^{b}$ with new HIV diagnosis & 1 & 1 & 0 \\
\hline & OPs re-linked to HIV care & 1 & - & - \\
\hline Gonorrhea/chlamydia & PrEP referrals accepted ${ }^{c}$ & 27 & 16 & 16 \\
\hline
\end{tabular}

Abbreviations: STD, sexually transmitted disease; PS, partner services; OP, original patient; DIS, disease intervention specialist; PrEP, HIV preexposure prophylaxis; N/A, not available

\footnotetext{
${ }^{a}$ Syphilis may include coinfections with GC and/or CT. GC may include coinfections with CT. CT refers to CT only infections.

${ }^{b}$ Clients refers to any STD PS cases, partners, or other contacts.

${ }^{c}$ Data for PrEP referrals accepted for King county includes OPs only and were estimated using empiric data. For Pierce and Spokane, PrEP referral data was collected via staff report for average weekly (Pierce) or quarterly (Spokane) numbers in 2018 and includes all STD PS client referrals (both cases and partners)
} 